\title{
The stability-plasticity dilemma: investigating the continuum from catastrophic forgetting to age-limited learning effects
}

\author{
Martial Mermillod ${ }^{1,2 *}$, Aurélia Bugaiska ${ }^{3}$ and Patrick Bonin ${ }^{2,3}$ \\ ${ }^{1}$ Centre National de la Recherche Scientifique, LPNC UMR 5105, Université Grenoble Alpes, Grenoble, France \\ 2 Institut Universitaire de France, Paris, France \\ ${ }^{3}$ LEAD-Centre National de la Recherche Scientifique, UMR 5022, University of Bourgogne, Dijon, France \\ ${ }^{*}$ Correspondence: martial.mermillod@upmf-grenoble.fr \\ Edited by: \\ Julien Mayor, University of Geneva, Switzerland \\ Reviewed by: \\ Michael Thomas, Birkbeck College, UK
}

The stability-plasticity dilemma is a wellknow constraint for artificial and biological neural systems. The basic idea is that learning in a parallel and distributed system requires plasticity for the integration of new knowledge, but also stability in order to prevent the forgetting of previous knowledge. Too much plasticity will result in previously encoded data being constantly forgotten, whereas too much stability will impede the efficient coding of this data at the level of the synapses. However, for the most part, neural computation has addressed the problems related to excessive plasticity or excessive stability as two different fields in the literature.

\section{THE PROBLEM OF CATASTROPHIC FORGETTING FOR DISTRIBUTED NEURAL NETWORKS}

The problem of catastrophic forgetting has emerged as one of the main problems facing artificial neural networks. The problem can be stated as follow: a distributed neural system, for example any biological or artificial memory, has to learn new inputs from the environment but without being disrupted by them. Catastrophic forgetting is defined as a complete forgetting of previously learned information by a neural network exposed to new information (McCloskey and Cohen, 1989; Ratcliff, 1990). This problem is a general problem that exists in different types of neural networks from standard back-propagation neural networks to unsupervised neural networks like self-organizing maps (Richardson and Thomas, 2008) or for connectionist models of sequence acquisition (Ans et al., 2002). Concerning artificial connectionist neural networks (such as, for instance, standard backpropagation networks), they are highly sensitive to catastrophic forgetting because of their highly distributed internal representations (French, 1992). Therefore, it is possible to reduce the problem of catastrophic forgetting by reducing the overlap among the internal representations stored in the neural network, for example using larger systems, or for example sparse or interleaved learning (Hetherington and Seidenberg, 1989; McRae and Hetherington, 1993). For this reason, when learning input patterns, connectionist networks have to alternate between them and adjust the corresponding synaptic weights by small increments in order to appropriately associate each input vector with the related output vector. By contrast, sequential learning in a standard connectionist network would result in the complete forgetting of previously learned input-output patterns. This problem affecting artificial neural networks clearly distinguishes them from the cognitive abilities of biological neural systems that are able to learn new patterns in sequential order without catastrophic forgetting.

In order to prevent catastrophic forgetting, various researchers have suggested using a dual-memory system which, fundamentally, simulates the presence of a short-term and a long-term memory (Robins, 1995; Ans and Rousset, 1997; French, 1997; Mermillod et al., 2003). The principle is to consolidate information, initially present in a short-term memory, within a long-term memory in order to prevent catastrophic forgetting in connectionist systems. This principle, investigated within the perspective of neural computation in artificial systems, could also point the way to a more general principle that also applies to biological neural systems (French, 1999).

\section{THE ENTRENCHMENT EFFECT: THE OPPOSITE EXTREME OF THE PLASTICITY-STABILITY DILEMMA}

At the opposite extreme of the stabilityplasticity continuum lies the entrenchment effect, which may contribute to age-limited learning effects (Zevin and Seidenberg, 2002; Bonin et al., 2004, 2009; Mermillod et al., 2009a). In the cognitive sciences, this research field emerged as part of the attempt to determine whether items which are acquired early in life are better memorized in adults than those which are acquired later in life. Various studies working within this perspective have shown that words acquired early are processed faster and more accurately than words acquired later in life (see Juhasz, 2005; Johnston and Barry, 2006 for reviews). These so-called age-of-acquisition effects have been found in a large variety of tasks, for example picture naming tasks, as well as in different populations (e.g., children and adults).

While distributed neural networks have long been used to address various issues in word recognition and spoken word production studies, they have also recently been used to investigate the computational basis of these age-limited learning effects (e.g., Ellis and Lambon Ralph, 2000; Zevin and Seidenberg, 2002; Lambon Ralph and Ehsan, 2006). In these connectionist models, lexical frequency is encoded in the strength of the connections between the different types of representations which 
are involved in recognizing and producing words (Seidenberg and McClelland, 1989; Plaut et al., 1996). As far as connectionist simulations of age-limited learning effects are concerned, Ellis and Lambon Ralph (2000) were the first to show that the order of introduction of the encounters determines the number of errors produced by the neural network at the end of training. More precisely, the items introduced first in their study produced fewer errors than the late-introduced items, even after cumulative frequency had been carefully controlled for. This effect of age-limited learning effects in connectionist networks is referred to as the entrenchment effect.

At a computational level, the question is to understand how this entrenchment effect emerges. According to Zevin and Seidenberg (2002), the loss of plasticity in connectionist networks such as Seidenberg and McClelland's (1989) was due to the adjustments of the weights that occur on the basis of the logistic function used by the backpropagation algorithm and permits adjustments to the weights (initially set to random values between 0 and 1). These adjustments are at their largest when the activations occur in the middle of the logistic function (around 0.5) and become smaller as the weights converge on values that cause unit activations to approximate more closely to the target values (for instance 1 or 0 ). Thus, there is a loss of plasticity (early trained patterns become entrenched in the weights) associated with the learning of the first patterns in the training regime. Therefore, according to Zevin and Seidenberg (2002), the loss of plasticity in connectionist systems should vary as a function of the transfer function and the error signal computed. For example, a root mean square vs. crossentropy error should produce different sensitivity to the entrenchment effect, but also to catastrophic forgetting. Of course, other factors as competition effects, loss of resources, and assimilation effects are important to produce age limited learning effects (Thomas and Johnson, 2006) and are important to control as possible confounded variables. In the current article, we suggest that the Fahlman offset (Fahlman, 1988) could constitute a simple and efficient way to test the computational basis of the loss of plasticity assumed by Zevin and Seidenberg (2002).

\section{THE FAHLMAN OFFSET: A WAY TO INVESTIGATE BOTH ENDS OF THE CONTINUUM}

It is interesting to note that the abovementioned research fields investigate two extremes of the same continuum. In other words, the entrenchment effect is related to a lack of plasticity (and an excess of stability) in response to newly acquired items, whereas catastrophic interference is related to an excess of plasticity (and a lack of stability) in response to new items presented sequentially. There are a number of ways of overcoming this difficulty, for instance by manipulating the orthogonality or the sparseness of the input-output patterns (French, 1992; Robins, 1995). However, among the different possibilities proposed to modulate the plasticity of neural networks, the method proposed by Fahlman (1988) is both simple and efficient. The basic idea is to add a constant number to the derivative of the sigmoid function (synaptic weights are adjusted by multiplying the error produced by a neuron by the derivative of the transfer function, i.e., the sigmoid function). This method makes it possible to avoid the entrenchment effect in the flat part of the sigmoid function and is relevant because this entrenchment effect is due to the flat spots at which the derivative of the sigmoid function approaches zero. Once the output value of a trained neural network starts to become entrenched around this flat spot of the sigmoid function, it becomes very difficult for the standard backpropagation algorithm to modify the synaptic weights responsible for producing this error. Even if an output value represents the maximum possible error, a unit whose output is close to 0.0 or 1.0 will be able to backpropagate only a tiny fraction of this error to the incoming weights and to units in earlier layers. Although it is theoretically possible to recover from entrenchment, this takes a very long time. The method proposed by Fahlman (1988), which consists of adding a small constant number to the derivative of the sigmoid function so that it does not go to zero for any output value, is therefore, both very simple and efficient to improve the efficiency of connectionist networks to simulate human cognitive processes (Mermillod et al., 2009b, 2010). For example, adding a constant of 0.1 to the sigmoid function before using it to scale the error prevents neuron values from approaching 0 and avoids the flat spots in the sigmoid function where the synaptic weights can become entrenched.

\section{NEW FINDINGS AND PERSPECTIVE}

In a recent article (Mermillod et al., 2012), we showed that age of acquisition can be considered, at a computational level, as an extreme case of frequency trajectory (i.e., the frequency with which a word is encountered during a certain period of life) and can help explain agelimited learning effects. Interestingly, no age-limited learning effects appeared when we used a Fahlman offset of 0.1 whereas it reappeared when we used a Fahlman offset of 0.0. This result was not consistent with Ellis and Lambon Ralph (2000) who reported an age-limited learning effect despite the improvement in the plasticity of the neural network brought about by modulating the Fahlman offset. This could be due to differences in the number or size of the training set between the two studies (Ellis and Lambon Ralph, 2000 or Mermillod et al., 2012). Therefore, the role of the training set in modulating the effects of learning parameters on age-limited learning and catastrophic interference remains a target of further investigation (since these factors could have a combined effect with neural plasticity). However, our results were not unambiguous: modifying the plasticity of an identical neural network by manipulating the Fahlman offset clearly modified the ability of the neural network to simulate (or not) age-limited learning effects. On the other side of the continuum, when the Fahlman constant was set to 0.0 , we observed the agelimited learning effects reported in the literature (Ellis and Lambon Ralph, 2000; Zevin and Seidenberg, 2002; Lambon Ralph and Ehsan, 2006). Moreover, one result that will surprise researchers working in the field of catastrophic forgetting is that this catastrophic forgetting effect was largely reduced after the period of entrenchment of synaptic weights (early acquired patterns for "adult" networks having been learnt at an early stage, compared to the medium and late patterns being learnt sequentially in a later stage). To conclude, we suggest here that investigating the plasticity-stability continuum by modulating the Fahlman offset 
should help us understand a wide range of cognitive phenomena from age-limited learning effects through to catastrophic forgetting, as well as various forms of memory disorders.

\section{ACKNOWLEDGMENTS}

This work was supported by a Institut Universitaire de France grant to Patrick Bonin and Martial Mermillod.

\section{REFERENCES}

Ans, B., and Rousset, S. (1997). Avoiding catastrophic forgetting by coupling two reverberating neural networks. C. R. Acad. Sci. III Sci. Vie 320, 989-997.

Ans, B., Rousset, S., French, R. M., and Musca, S. (2002). "Preventing catastrophic interference in multiple-sequence learning using coupled reverberating elman networks," in Proceedings of the 24th Annual Meeting of the Cognitive Science Society, eds W. D. Gray and C. D. Shunn (Mahwah, NJ: Lawrence Erlbaum Associates).

Bonin, P., Barry, C., Méot, A., and Chalard, M. (2004). The influence of age of acquisition in word reading and other tasks: a never ending story. J. Mem. Lang. 50, 456-476.

Bonin, P., Méot, A., Mermillod, M., Ferrand, L., and Barry, C. (2009). The effects of age of acquisition and frequency trajectory on object naming. Q. J. Exp. Psychol. 62, 1-9.

Ellis, A. W., and Lambon Ralph, M. A. (2000). Age of acquisition effects in adult lexical processing reflect loss of plasticity in maturing systems: insights from connectionist networks. J. Exp. Psychol. Learn. Mem. Cogn. 26, 1103-1123.

Fahlman, S. E. (1988). "Faster-learning variations on back-propagation: an empirical study," in Proceedings of the 1988 Connectionist Models Summer School, eds D. S. Touretzky, G. E. Hinton, and T. J. Sejnowski (Los Altos, CA: Morgan Kaufmann), 38-51.

French, R. M. (1992). Semi-distributed representations and catastrophic forgetting in connectionist networks. Connect. Sci. 4, 365-377. doi: 10.1080/09540099208946624

French, R. M. (1997). Pseudo-recurrent connectionist networks: an approach to the "sensitivitystability" dilemma. Connect. Sci. 9, 353-379. doi: 10.1080/095400997116595
French, R. M. (1999). Catastrophic forgetting in connectionist networks. Trends cogn. Sci. 3, 128-135. doi: 10.1016/S1364-6613(99)01294-2

Hetherington, P. A., and Seidenberg, M. S. (1989) "Is there 'catastrophic interference' in connectionist networks?" in Proceedings of the 11th Annual Conference of the Cognitive Science Society, (Hillsdale, NJ: Erlbaum), 26-33.

Johnston, R. A., and Barry, C. (2006). Age of acquisition and lexical processing. Vis. Cogn. 13, 789-845.

Juhasz, B. (2005). Age-of-acquisition effects in word and picture identification. Psychol. Bull. 131, 684-712. doi: 10.1037/0033-2909.131.5.684

Lambon Ralph, M. A., and Ehsan, S. (2006). Age of acquisition effects depend on the mapping between representations and the frequency of occurrence: empirical and computational evidence. Vis. Cogn. 13, 884-910.

McCloskey, M., and Cohen, N. J. (1989). Catastrophic interference in connectionist networks: the sequential learning problem. Psychol. Learn. Motiv. 24, 109-165. doi: 10.1016/S0079-7421(08)60536-8

McRae, K., and Hetherington, P. (1993). "Catastrophic interference is eliminated in pretrained networks," in Proceedings of the $15 \mathrm{~h}$ Annual Conference of the Cognitive Science Society, (Hillsdale, NJ: Erlbaum), 723-728.

Mermillod, M., Bonin, P., Méot, A., Ferrand, L., and Paindavoine, M. (2012). Computational evidence that frequency trajectory theory does not oppose but emerges from age of acquisition theory. Cogn. Sci. 36, 1499-1531. doi: 10.1111/j.15516709.2012.01266.x

Mermillod, M., Bonin, P., Mondillon, L., Alleysson, D., and Vermeulen, N. (2010). Coarse scales are sufficient for efficient categorization of emotional facial expressions: evidence from neural computation. Neurocomputing 73, 2522-2531. doi: 10.1016/j.neucom.2010.06.002

Mermillod, M., Bonin, P., Morisseau, T., Méot, A., and Ferrand, L. (2009a). "Frequency trajectory gives rise to an age-limited learning effect as a function of input-output mapping in connectionist networks," in Proceedings of the 31th Annual Conference of the Cognitive Science Society, eds N. Taatgen and H. van Rijn (Mahwah, NJ: Lawrence Erlbaum Associates), 2322-2327.

Mermillod, M., Vermeulen, N., Lundqvist, D., and Niedenthal, P. M. (2009b). Neural computation as a tool to differentiate perceptual from emotional processes: the case of anger superiority effect. Cognition 110, 346-357. doi: 10.1016/j.cognition.2008.11.009
Mermillod, M., French, R. M., Quinn, P. C., and Mareschal, D. (2003). "The importance of longterm memory in infant perceptual categorization," in Proceedings of the 25th Annual Conference of the Cognitive Science Society, eds R. Alterman and D. Kirsh (Mahwah, NJ: Lawrence Erlbaum Associates), 804-809.

Plaut, D. C., McClelland, J. L., Seidenberg, M. S., and Patterson, K. (1996). Understanding normal and impaired reading: computational principles in quasi-regular domains. Psychol. Rev. 103, 56-115. doi: 10.1037/0033-295X.103.1.56

Ratcliff, R. (1990). Connectionist models of recognition memory: constraints imposed by learning and forgetting functions. Psychol. Rev. 97, 285-308. doi: 10.1037/0033-295X.97.2.285

Richardson, F., and Thomas, M. S. C. (2008). Critical periods and catastrophic interference in selforganising feature maps. Dev. Sci. 11, 371-389. doi: 10.1111/j.1467-7687.2008.00682.x

Robins, A. (1995). Catastrophic forgetting, rehearsal, and pseudorehearsal. Connect. Sci. 7, 123-146. doi: 10.1080/09540099550039318

Seidenberg, M. S., and McClelland, J. L. (1989). A distributed, developmental model of word recognition and naming. Psychol. Rev. 96, 523-568. doi: 10.1037/0033-295X.96.4.523

Thomas, M. S., and Johnson, M. H. (2006). The computational modeling of sensitive periods. Dev. Psychobiol. 48, 337-344. doi: 10.1002/dev.20134

Zevin, J. D., and Seidenberg, M. S. (2002). Age of acquisition effects in word reading and other tasks. J. Mem. Lang. 47, 1-29. doi: 10.1006/jmla.2001.2834

Received: 29 April 2013; accepted: 17 July 2013; published online: 05 August 2013.

Citation: Mermillod M, Bugaiska A and Bonin P (2013) The stability-plasticity dilemma: investigating the continuum from catastrophic forgetting to age-limited learning effects. Front. Psychol. 4:504. doi: 10.3389/ fpsyg.2013.00504

This article was submitted to Frontiers in Language Sciences, a specialty of Frontiers in Psychology.

Copyright (C) 2013 Mermillod, Bugaiska and Bonin. This is an open-access article distributed under the terms of the Creative Commons Attribution License (CC BY). The use, distribution or reproduction in other forums is permitted, provided the original author(s) or licensor are credited and that the original publication in this journal is cited, in accordance with accepted academic practice. No use, distribution or reproduction is permitted which does not comply with these terms. 